\title{
Basic considerations on seasonal breeding in mammals including their testing by comparing natural habitats and zoos
}

\author{
Marcus Clauss $^{1}$ (1) $\cdot$ Philipp Zerbe ${ }^{1} \cdot$ Laurie Bingaman Lackey $^{2} \cdot$ Daryl Codron $^{3} \cdot$ Dennis W. H. Müller $^{4}$
}

Received: 24 August 2020 / Accepted: 7 October 2020 / Published online: 21 October 2020

(c) The Author(s) 2020

\begin{abstract}
Seasonal reproduction is common in mammals. Whereas specific conditions triggering a seasonal response can only be identified in controlled experiments, large-scale comparisons of reproduction in natural habitats and zoos can advance knowledge for taxa unavailable for experimentation. We outline how such a comparison can identify species whose seasonal physiology is linked to photoperiodic triggers, and those whose perceived seasonality in the wild is the consequence of fluctuating resources without a photoperiodic trigger. This concept groups species into those that do not change their aseasonal pattern between natural habitats and zoos because they are not constrained by resources in the wild, those that do not change a seasonal pattern between natural habitats and zoos because they are triggered by photoperiod irrespective of resources, and those that change from a more seasonal pattern in the natural habitat to an aseasonal pattern in zoos because the zoo environment alleviates resource limitations experienced in the wild. We explain how detailed comparisons of mating season timing in both environments can provide clues whether a specific daylength or a specific number of days after an equinox or solstice is the likely phototrigger for a taxon. We outline relationships between life history strategies and seasonality, with special focus on relative shortening of gestation periods in more seasonal mammals. Irrespective of whether such shortening results from the adaptive value of fitting a reproductive cycle within one seasonal cycle (minimizing 'lost opportunity'), or from benefits deriving from separating birth and mating (to optimize resource use, or to reduce infanticide), reproductive seasonality may emerge as a relevant driver of life history acceleration. Comparisons of data from natural habitats and zoos will facilitate testing some of the resulting hypotheses.
\end{abstract}

Keywords Reproduction $\cdot$ Breeding $\cdot$ Seasonality $\cdot$ Body condition $\cdot$ Life history $\cdot$ Zoo

\section{Introduction}

Many mammals are subject to seasonal fluctuations in their environment. Seasonal changes affecting food availability and convective temperature loss determine the animal's

Handling editor: Yoshiyuki Henning.

Marcus Clauss

mclauss@vetclinics.uzh.ch

Philipp Zerbe

philipp.zerbe@gmx.net

Laurie Bingaman Lackey

giraffe3@bellsouth.net

Daryl Codron

CodronD@ufs.ac.za

Dennis W. H. Müller

dennis.mueller@zoo-halle.de energy budget (Bronson 2009). Among the various adaptations to these fluctuations, seasonal reproduction plays a central role (Bronson 1989; Bronson and Heideman 1994) to ensure the survival of the offspring and (lactating) mothers. Many crucial hormonal and genetic mechanisms linked to seasonal reproduction have been elucidated in selected species by detailed experimental investigations

1 Clinic for Zoo Animals, Exotic Pets and Wildlife, Vetsuisse Faculty, University of Zurich, Zurich, Switzerland

2 World Association of Zoos and Aquariums (WAZA), Carrer de Roger de Llúria, 2, 2-2, Barcelona, Spain

3 Department of Zoology and Entomology, University of the Free State, Bloemfontein, South Africa

4 Zoological Garden Halle (Saale), Fasanenstr. 5a, 06114 Halle (Saale), Germany 
(reviewed e.g. by Bronson 2009). But for a large-scale understanding of the phenomenon across mammalian species and clades, where experimental work is logistically out of the question, comparative approaches are required. Publications reporting on such studies are, in our opinion, sometimes conceptually less stringent than those reporting on experimental work. While not offering a comprehensive review, it is our aim in the current contribution to provide a set of concepts that can be applied to, and partially also tested by, comparative studies, and that may add clarity to the approach to seasonal reproduction.

Reproductive seasonality has been studied from various angles - from that of the evolutionary ecologist, the physiologist, the chronobiologist, or the molecular geneticist (Visser et al. 2010). We adopt the angle of comparative zoologists working with animals in zoos. We first offer a simple concept about distinguishing condition-threshold-controlled reproduction and photoperiod-induced reproduction, touching on putative conditions in different natural habitats, stressing differences between regularity and predictability. We then address issues of what kinds of photoperiodic signals may be transduced as trigger for breeding activity. Finally, we discuss selected life history components that may be linked to seasonal reproduction. In all these sections, we particularly emphasize how using data from animals kept in zoos, when compared to reproduction in the natural habitats, can help in testing the resulting hypotheses.

Even though the following point may be well known to most readers, it may be prudent to remind us that when dealing with seasonal reproduction, the data most easily observed and used are that of births, although mating behavior might also be an option. Births are not controlled per se, and mammals have, with only a few exceptions, very limited capacity to accelerate or delay the in utero development speed of their embryos, or the birth of term offspring. Rather, it is the timing of breeding that determines, together with the species-specific gestation period (and, possibly, periods of delayed implantation), the date of birth. The ultimate cause for seasonal reproduction is most likely the timing of births in relation to favorable environmental conditions. Favorable condition ensures sufficient food resources for the lactating mothers and those newborns that ingest solid food, and thermic conditions that support survival of the offspring. The proximate cause, however, cannot be a triggering of birth by any external or internal factors, but the triggering of mating. In other words, if one hypothesizes that a certain environmental cue, for example, photoperiod, temperature, or humidity, controls the seasonality of reproduction, one has to explain the status of this cue at the time of, and how it might trigger, breeding activity.

\section{Body condition-threshold-controlled reproduction}

Bronson (2009) stated that in all animals, irrespective of whether they live in seasonal or non-seasonal environments, reproduction is controlled by body condition. Nevertheless, comparative approaches often conceptualize a dichotomy between photoperiod-controlled and condition-controlled seasonality, giving rise to the assumption that both are adaptations that evolved for seasonal reproduction (e.g., Brockman and van Schaik 2005; Ogutu et al. 2010). Following Bronson (2009), we argue that this dichotomy is not warranted. Rather, condition-control of reproduction is a fundamental, underlying physiological mechanism common to all mammals (with few exceptions, see below) (Celik et al. 2015; D'Occhio et al. 2019). Irrespective of the environmental conditions, if body condition is below a certain threshold, conception (or maintenance of pregnancy) may be prevented. If the condition is above the threshold (but below the magnitude of pathological obesity), conception (or maintenance of pregnancy) is facilitated. The physiological mechanism itself has no predictive power as to whether a favorable season will follow or not.

The condition-controlled threshold may be considered a general safety mechanism ensuring minimal conditions (Owen-Smith 1988, p. 184), an 'emergency break' (Zerbe et al. 2012; Ogutu et al. 2015) or a 'right now, we are fine-so let's begin (or continue) and see what happens'-signal. So to speak, condition-controlled threshold mechanisms focus on the survival of the mothers as prerequisite to raise viable offspring. It protects the animal from making unwise investments, irrespective of whether the reasons for being below the permissive threshold are totally fortuitous, as in the case of disease, or regular, as in seasonally fluctuating environments. This mechanism will be the main determinant of reproductive biology, and, therefore, a focus of attention, in species that did not evolve dependence on a photoperiod zeitgeber.

In theory, mammals could differ in the magnitudes of body condition threshold that control reproduction, with some species requiring a comparatively higher body condition than others, e.g., in terms of the proportion of the body that must be represented by adipose tissue. To our knowledge, no comparative investigation on the topic exists.

The condition-threshold mechanism will lead to different reproductive patterns in different environments (Fig. 1).

\section{Unconstrained environments}

It is a reasonable assumption that the average zoo husbandry regime will always provide animals with sufficient 


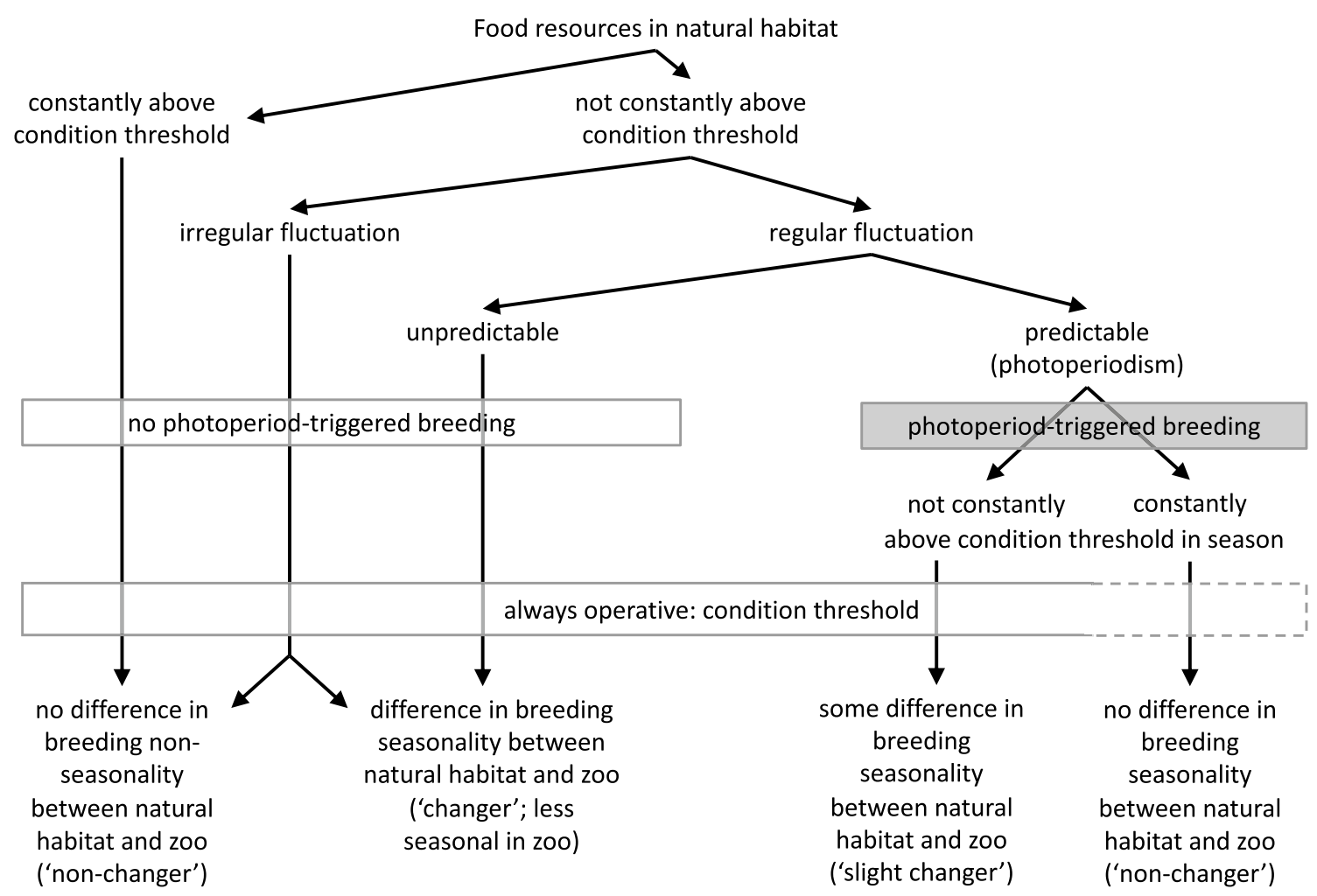

Fig. 1 Simplified decision tree of how environmental conditions in natural habitats differ between mammals of different degrees of reproductive seasonality, and how the reproduction of these mammals will generally appear under zoo conditions. Note that in this scheme, the body condition-threshold-related control of reproduction is always thought to be operative (with a potential exception in some, but not

energy resources, without imitating natural conditions by creating times of energy deficit. The question why few zoological institutions actively attempt to imitate natural conditions in this respect, even though this has been repeatedly advocated (e.g., Lechner-Doll et al. 2000; Clauss et al. 2003), is not discussed here. To our knowledge, the only reasonable objection to considering zoos as environments with a constant provision of sufficient resources and opportunities for reproduction is the case of large species, such as elephants or rhinoceroses, that share a peculiar set of characteristics: In these species, animals of opposite sex often cannot be kept together at all times due to their specific social structures; these species cannot be kept outside for longer periods during the colder parts of the temperate zone winter; and they may require sufficient space for safe matings. In zoos of the temperate zone, the necessary space may not be available in indoor facilities, and therefore, matings may be artificially reduced during a certain time of the year. For this reason, these large species should receive special consideration when assessing reproductive seasonality in zoos (Hufenus et al. 2018). all, photoperiod-controlled mammals). Note that animals that do not differ in reproductive seasonality between their natural habitat and zoos (the so-called 'non-changers') appear on both extremes of the spectrum-those 'non-changers' that are non-seasonal in both habitats, and those 'non-changers' that are seasonal in both habitats

But given the assumption of unlimited resource provision in zoos, it is to be expected that more mammals show seasonal reproduction in natural habitats (where resource availability may be periodically limited so that body condition is below the reproduction-controlling threshold) than in zoos. Thus, the primary distinction between natural habitats is whether they never put the animal into a body condition below the reproduction-controlling threshold, or whether conditions below that threshold do occur (Fig. 1). The finding that a species does not reproduce seasonally, either in the wild or in captivity, is not conclusive evidence that the natural environment offers unconstrained resources-for that, a thorough habitat evaluation would be required. However, such a finding provides parsimonious circumstantial evidence.

\section{Constrained environments: irregular or regular?}

While condition-controlled reproduction is ubiquitous, the question of whether it leads to a detectable pattern of seasonal reproduction depends on whether the fluctuations leading to temporal conditions below the threshold are regular 
or not. Evidently, irregular fluctuations will affect breeding, but not in a pattern that is consistent over time and hence detectable as seasonality. In this way, environments with irregular resource fluctuations may resemble environments without resource fluctuations in the reproductive patterns of the animals living in them (Fig. 1). This mismatch may result in reduced survival of offspring when compared to environments with regular, predictable fluctuations. Alternatively, especially if the period of observation in the natural habitat does not span many annual cycles, these irregular fluctuations may stochastically lead to the impression of some reproductive seasonality, an impression that will not be similar in specimens kept in zoos. Important examples of strong, irregular fluctuations are the El Niño effect, or fruit mast years.

Environments with regular fluctuations, on the other hand, will lead to reproductive patterns of regular fluctuations, with mating seasons at times where body condition allows conception, and birthing season after the time corresponding to the gestation period. In certain environments, the moment where a threshold condition is reached and conceptions begin may represent the onset of a longer period of resource availability, such as in mast years or a general fruiting season (van Schaik and van Noordwijk 1985; Kanamori et al. 2010). And if gestation periods are of a duration that allow births in this same period, the condition-threshold mechanism might be interpreted to have evolved as a particular adaptation to habitat seasonality. But the fact that animals reacting to this mechanism are rather characterized by longer rather than shorter gestation periods (Heldstab et al. 2020) that would make coinciding of births in the same period more likely, speaks against this interpretation.

Regular, or in other words seasonal, patterns raise the question of how they are triggered, and how we can determine the trigger. Again, given the enormous logistical effort required for experimental work, the comparison with zoo animals comes in handy. If zoo specimens have a less distinct seasonal reproduction or are non-seasonal compared to free living ones, specimens in natural habitats can be assumed to respond to the condition-threshold mechanism. If zoo specimens show a similar seasonal reproduction in spite of the constant resource provision in zoos, then the conclusion that the species' reproduction is triggered by an additional signal, particularly a photoperiodic one, is parsimonious.

These two possibilities, in turn, raise the question of what the decisive factor for each of them is.

\section{Environments with regular fluctuations: unpredictable or predictable?}

From an anthropocentric standpoint, making the distinction between predictable and unpredictable regularity may seem superfluous. For us, predictability is the ultimate test for calling a pattern 'regular', and we use calendars or calendrical memory to detect regularity. Being unable to use calendars, however, animals have to rely on environmental cues that facilitate the prediction of seasons.

The most reliable regular environmental information is photoperiod. If environmental conditions are fluctuating regularly, and the environment also provides a regularly fluctuating photoperiodic signal, then a photoperiod-dependent control mechanism for reproduction can evolve. The fact that the tilt of the earth's rotational axis is responsible for both regular fluctuations in environmental temperatures and photoperiod leads to a very high reliability of the photoperiodic signal. Species with photoperiod-controlled reproduction are likely to maintain their reproductive seasonality when kept in zoos at latitudes that provide detectable photoperiodic cues. This has been shown repeatedly in various mammal groups (demonstrated and reviewed in Zerbe et al. 2012; Heldstab et al. 2018, 2020).

But seasonal differences in photoperiod are not of similar distinctiveness across all habitats. In tropical habitats, especially close to the equator below latitudes of $11.75^{\circ}$ (Bronson and Heideman 1994), seasonal differences in photoperiod are so slight that they are deemed unsuitable as seasonal clues. Nevertheless, environments in these regions may still be characterized by regular fluctuations, such as the alteration between rainy and dry seasons. These habitats are, from a human point of view, regular, but from an animal point of view, unpredictable. Unless the species living in these environments evolved at higher latitudes and have only occupied these tropical habitats more recently, possibly still bearing remnants of a photoperiodic trigger from their historical habitats (Hufenus et al. 2018), we would not expect them to respond to photoperiodic cues but reproduce only under control of the condition-threshold mechanism. Hence, they should be less seasonal under constant resource provision in zoos.

\section{Environments with predictable fluctuations: predictable temporal onset and additionally predictable resource magnitude?}

When referring to the 'predictability' of an environment, it is crucial to define whether this predictability refers to the variation in the magnitude of resource availability between years, or the temporal onset of a change in resources. The onset of seasons may be very predictable, being linked to solstices and equinoxes. The magnitude of environmental change linked to the seasons, by contrast, may be less predictable, because photoperiodic cues alone cannot foretell whether a winter will be harsh or mild.

The concept of resource predictability has been applied in both meanings in investigations of seasonal reproduction. 
For example, Blanco et al. (2015) determined a low predictability of the amount of seasonal resources available in Madagascar. Without making the distinction between the temporal predictability of seasons and the predictability of the magnitude of the seasonal resources, the concept that species with a photoperiod-controlled seasonal reproduction live in 'highly unpredictable environments' (Blanco et al. 2015) appears self-contradictory. Madagascar, while located in the tropics, is home to a large number of highly seasonal species (Heldstab et al. 2020). Whether Madagascar, or any other particular habitat, is peculiar due to a higher regularity in the temporal onset of the seasonal change of resources than other tropical seasonal environments, remains to be documented in this context.

Depending on the characteristics of the natural habitat, different interactions between photoperiod and conditionthreshold control mechanisms may evolve. On the one hand, the condition-threshold mechanism may still prevent conception even though the photoperiod trigger may have initiated a general 'permissive state' for reproductive activity. This may play a role in natural environments where the availability of the seasonal resources may vary to a higher degree between different years, with a more restricted seasonal reproduction than in zoos, where the full potential of the photoperiodically permissive period can be used.

On the other hand, if predictable regularity is high and seasonal resource availability is always sufficient for the species in question, but conception takes place prior to resource acquisition for late pregnancy and lactation, species may evolve very low condition thresholds (Brockman and van Schaik 2005). In other words, some species may evolve to not use the condition-threshold mechanism due to the overruling predictability of their environment (Fig. 1). The more stable the resource availability in the peak season of a predictable habitat, the more likely a seasonal species may be to lose, or evolve fewer, condition-threshold mechanisms. Such a reliability of resources may be more likely for smallersized species with lower absolute requirements, as in the example of the mouse lemur (Microcebus spp.) (Brockman and van Schaik 2005).

\section{Photoperiod-controlled reproduction}

The neuroendocrinological physiology underlying the triggering of reproductive function by photoperiod has been well described (reviewed, e.g., by Bronson and Heideman 1994; Bronson 2009). The general concept is that animals rely on endogenous rhythms or 'timers' that are on the one hand constantly adjusted by external, photoperiodic cues, and that on the other hand use specific photoperiodic signals as triggers (Prendergast 2005; Paul et al. 2008; Bronson 2009). In theory, as outlined by Bronson and Heideman
(1994), these triggers can be of different kinds - an immediate release signal (as in: a certain photoperiodic cue triggers the initiation of activity) or an indirect release signal (as in: a certain photoperiodic cue sets off an internal timer that then 'counts down' until the initiation of activity), or, in a similar manner, immediate or indirect cessation signals. Evidently, in animals that breed seasonally, not only an initiating but also a cessation signal is required. Often, this seems to be linked to an internal timer that induces 'refractoriness' to the triggering signal (Prendergast 2005; Paul et al. 2008; Bronson 2009). Given the variety of gestation periods (that necessitate photoperiodic signals at different times of the year to result in birth during the period of optimal conditions), the multitude of potential photoperiodic cues, and possibly of the internal timers, a large number of detailed adaptations appear possible.

Animals with photoperiod-controlled reproduction are typically grouped into 'short-day' or 'long-day' breeders, depending on the tendency of the daylength change during their mating season (Hansen 1985). Most seasonally reproducing animals have been defined in this way. However, what determines the exact timing of the onset of the mating season within these time periods (that each last half a year) is less well known. Experimental approaches are often set up to trigger reproductive activity by changing daylength and measuring the corresponding hormonal, behavioral and anatomical changes, but not to detect the exact natural photoperiodic signal necessary for the triggering. Among the possible options, two stand out that should lead to clearcut signals when comparing the patterns of reproduction between natural habitats and zoos-if the zoos are located, individually or on average, at a latitude different from the natural habitat.

The beginning of the mating period might be determined by an internal clock that tracks the number of days after a certain specific event, such as a solstice or an equinox (Fig. 2a). This is a situation that has been tentatively described for primates (Heldstab et al. 2020). Such a mechanism has also been reported for domestic sheep (Robinson et al. 1985; Nicholls et al. 1989) and goats (Delgadillo et al. 2011) exposed to different experimental light regimes.

Alternatively, the trigger of the mating period might be, after a general cueing of the daylength change (as, e.g., shown in roe deer Capreolus capreolus by Sempéré et al. 1995), the absolute day length, which is - unless it coincides exactly with the equinox-reached at different latitudes at different Julian days of the year (Fig. 2b). This is a situation that has been tentatively described for nondomestic ruminants (Zerbe et al. 2012), in contrast to the findings in domestic small ruminants mentioned above. In this scenario, the direction of day length change (i.e., days becoming shorter) 'sets the stage' of animals being generally receptive to the detailed triggering of the absolute day length. 


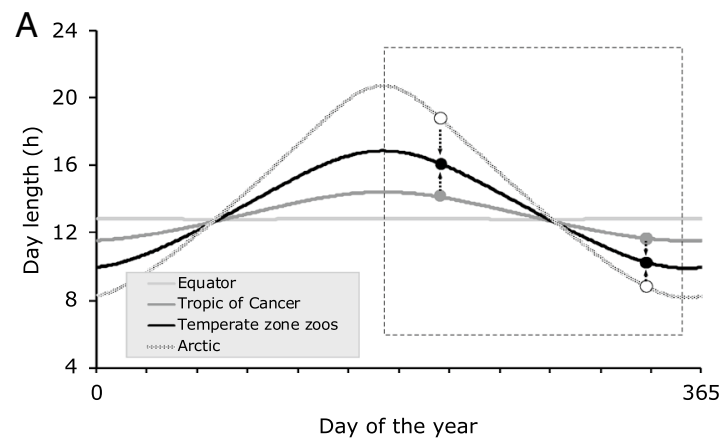

Fig. 2 Possible scenarios for systematic changes of the beginning of the mating season within a species kept at different latitudes (for example, in zoos). The examples display short-day breeders (with a theoretical window for the initiation of mating in the second half of the year), but the principles apply to long-day breeders as well. a Within the respective half of the year, the absolute number of days (after the solstice, or after equinox) is the trigger, leading to systematic shifts of the day length at the onset of mating activity but to iden-

These previous analyses have been constrained by the fact that average latitudes were assumed for zoos, rather than analyzing zoo data separately by latitude of the holding institutions. The latter would be a valuable approach to further characterize individual species' photoperiodic physiology. The failure to detect such a pattern, for example within the Carnivora (Heldstab et al. 2018), could be due to the fact that other, yet-to-be-considered photoperiodic trigger mechanisms exist in mammals, or that within the phylogenetic group investigated, a variety of mechanisms exist that result in no clear overall result. The fact that endogenous clock rhythms and timers exist in mammals that often, but not always, use photoperiodic cues for calibration, opens possibilities for a large variety of theoretical combinations of photoperiodic signal, endogenous clocks, and conditionthreshold mechanisms.

\section{Other triggers for seasonal reproduction}

Apart from photoperiodic cues, other triggers may exist, deriving from abiotic or biotic factors, such as humidity, social cues, or specific plant compounds (reviewed, e.g., by Paul et al. 2008). Such triggers are typically species specific. They have been specifically invoked in the forecasting of mast years and initiation of reproductive activity to time births with optimal resource conditions (reviewed in Tissier et al. 2020). In theory, such triggers could be implied in situations where breeding in a zoo setting does not occur satisfactorily, seemingly lacking a specific cue. Such triggers are unlikely to explain large-scale, multi-species comparative patterns between natural habitats and zoos, but may be important for specific species. Such signals might not only

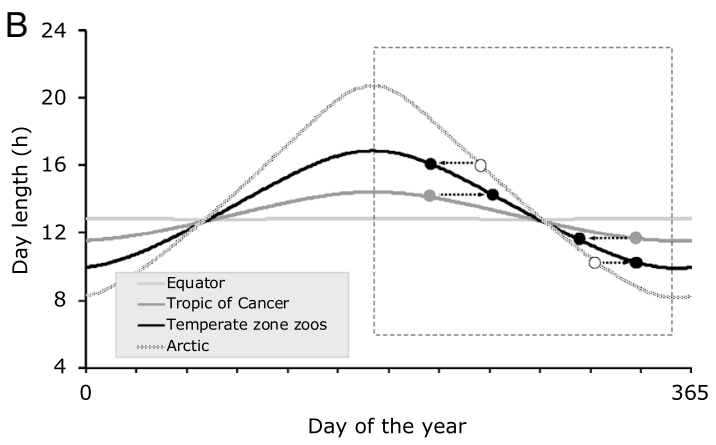

tical Julian days across latitudes. b Within the respective half of the year, the absolute daylength is the trigger, leading to systematic shifts in the Julian day of the onset of mating activity; the direction of the shift depends on the latitude of origin and zoo, and the position of the absolute daylength in relation to the equinox. A situation similar to a has been described in primates (Heldstab et al. 2020), and a situation similar to b in ruminants (Zerbe et al. 2012)

be relevant for primary consumers, but also possibly for secondary consumers. For example, while it is known that owls produce a higher number of surviving fledglings in mast years (Lithner and Jonsson 2002; Solonen 2005), this is typically explained by the higher availability of prey during these years. The senior author, however, made the observation that breeding pairs of Strix uralensis kept in aviaries of a reintroduction program, and provided with consistent care and feeding over years, produced more surviving offspring in mast years (D.W.H. Müller, personal observation).

In particular, because the condition-threshold mechanism will make animals in resource-limited habitats responsive to the onset of a 'rainy season' with increased resource availability, the conclusion that the species might have evolved a humidity cueing can be appealing. In the absence of experimental testing of the hypothesis (e.g., by exposing animals at constant resource conditions to varying degrees of humidity), a reaction to a condition-threshold mechanism rather than a humidity-cued triggering of reproductive activity appears the more parsimonious explanation.

\section{Seasonal reproduction and life history}

Seasonal reproduction has been linked to a variety of life history characteristics in mammals. Here, we first discuss seasonality in relation to potential misconceptions about 'income' and 'capital' breeding strategies. Then, we compare different concepts on the relationship between seasonality and the complex of the related factors of body size, longevity and the pace of life history. Lastly, we expand on the observation that more seasonal eutherian mammals 
apparently are characterized by comparatively short gestation periods for their body size.

\section{Seasonality and the 'income' and 'capital breeding' dichotomy}

The definition of a 'capital breeder' is 'an organism that uses stored energy for reproduction', as opposed to an 'income breeder' that 'uses energy acquired during the reproductive period [...] for reproduction' (Stearns 1992, p. 221-2). In many mammals, both body stores and newly ingested food will be used during the reproductive period, making a clear distinction impossible for most species. Most mammals will continue feeding during peak pregnancy and lactation, and hence be 'income breeders' to some extent; many mammals will also lose some body mass during lactation, and hence also be 'capital breeders' to a certain extent. For a classification of mammals on this continuum, the proportions of energy derived from stores built up prior to the reproductive period, and from food ingested during this period, would need to be assessed. During the lactation period, a comparison of the weight loss of the mother and the weight gain of the offspring might be a useful proxy. To our knowledge, no corresponding comparative, quantitative investigation in mammals exists.

For individual species comparisons, such as between muskoxen (Ovibos moschatus) as 'more of a capital breeder' and reindeer (Rangifer tarandus) as 'more of an income breeder', the graduality of the transition is evident (Kerby and Post 2013). Only in those species that experience such a distinct spatial separation between the location of birth and lactation on the one hand and their feeding grounds on the other, so that they fast completely during lactation (and possibly late gestation), can a truly 'capital breeder' strategy be assigned, e.g., in certain pinnipeds, ursids, or cetaceans.

Sometimes, the difference between photoperiod-controlled and condition-threshold-controlled reproduction has been linked to the dichotomy developed in behavioral ecology between 'capital' and 'income breeding' (Brockman and van Schaik 2005; Janson and Verdolin 2005; Lee et al. 2017). However, we agree with Lewis and Kappeler (2005) and Stephens et al. (2009) that there is no direct link between these two dichotomies. This link should, therefore, be reconsidered, not because it would amount to equating distinct photoperiod-controlled seasonality with income breeding, but mainly due to the equating of an absence of photoperiod control with 'capital breeding'. This is conceptually problematic because the body reserves necessary to surpass the condition threshold are not, in their magnitude, comparable to the 'capital' that would be required to support late gestation or lactation without additional resource intake. In particular, animals with multi-year reproductive cycles, such as orangutans (van Noordwijk et al. 2013), cannot be 'capital breeders' - they evidently will fuel requirements during these long periods much more by the respective income than by a priori body stores.

Rather than equating the terms 'income' and 'capital breeding' with the presence and absence of photoperiod control, the distinction is applied most fruitfully within species with photoperiodic control. For example, in pinnipeds, which are highly seasonal breeders (Heldstab et al. 2018), the two distinct breeder types, 'income' or 'capital', are defined with no reference to the degree of seasonality as such (Boyd 2000). Note that by definition, 'capital breeders' do not necessarily acquire their 'capital' prior to the decision to initiate reproduction, i.e., prior to conception-they acquire it prior to needing it, with the main requirement, in mammals, being in the lactation stage.

Assessing whether a mammal in an unpredictable environment is a 'capital' or an 'income' breeder appears of lesser interest. In contrast to the general statement of Jönsson (1997), a mainly 'capital breeding' strategy appears of little value under unpredictable conditions for mammals, because the decision to initiate reproduction, i.e., to mate, is at least a gestation period away from the time when the capital is needed. True mammal 'capital breeding' in unpredictable environments, i.e., where the animal cannot rely on the time between conception and birth to suffice for capital acquisition, would, hence, require the accumulation of stores that last long enough prior to mating. To delay breeding until such stores are built up, if that is even possible, appears to provide little advantage over a predominantly 'income breeding' strategy that continuously adjusts for current conditions using the condition-threshold mechanism.

\section{Seasonality, body size, longevity and lost opportunity}

\section{The special case of very small mammals}

Bronson $(1989,2009)$ proposed a model that distinguished small mammals with a life expectancy of about half a year from larger mammals with life expectancies $>1$ year. According to this model, larger mammal species should start becoming photoperiod-controlled at the lowest possible latitudes, and from the tropics of Cancer or Capricorn onwards should always have evolved photoperiod control. By contrast, very small mammal species with short lifespans can comprise two phenotypes-one that is photoresponsive, and another that is not but that only depends on the condition-threshold mechanism. How many small mammals show these two phenotypes is, to our knowledge, unknown; however, Bronson (2009) suggested that it should occur in all species above a latitude of $40^{\circ}$. Additionally, photoperiod control starts at higher latitudes in smaller mammals (Bronson 1989, 2009). At the short time scales relevant for the 
reproduction of these small species, favorable conditions may occur irrespective of the overarching annual cyclicity. The reliance on both kinds of phenotypes, that in sum will use the predictably favorable seasonal conditions and additionally any spontaneously occurring favorable time windows, is, therefore, a valid evolutionary adaptation that ensures no opportunities are lost for the species. In zoos, such species should, hence, be generally less seasonal than in their natural habitats, even though photoperiod control is operative in certain individuals.

\section{Is reproductive seasonality not adaptive in mammals with long lactation periods?}

For larger mammals with comparatively slow life histories and long lactation periods spanning more than one year, including great apes (Gorilla, Pan, Pongo), giraffes (Giraffa camelopardalis) or elephants (Elephas, Loxodonta), van Noordwijk et al. (2013) argued that no specific timing of mating in relation to resource fluctuations should occur. They argue that the comparatively low additional food requirements of lactating mothers as compared to the non-lactating maintenance state would make the benefits of seasonal reproduction too low to outweigh the costs of lost breeding opportunity, suggesting that these species should rather be in a "permanent reproductive state". This view might appear problematic for several reasons. (i) It focuses on absolute time lengths ( $>1$ year) rather than on the question whether these long times are in synch or out of synch with an annual cycle (i.e., whether they are multiples of 1 year or not). (ii) It focuses solely on the resources required by lactating mothers but not on effects of environmental conditions on neonate survival. (iii) It would require that test cases of large mammals with long lactation periods that live or lived in temperate environments (such as Bactrian camels Camelus bactrianus in the Mongolian desert, or mammoths in the steppe-tundras of the northern hemisphere) show no evidence for seasonal reproduction.

(i) Kiltie (1984) explained that the disadvantage of having a fixed reproductive cycle out of synch with the annual cycle, in terms of 'lost opportunity' or time necessarily spent waiting before the next reproductive cycle, is higher for hypothetical species that just 'barely miss' the annual cycle (e.g., have a reproductive cycle of 13 or 25 months), but decreases continuously with the length of the cycle (e.g., the proportion of 'time lost' due to a fixed, out-of-synch reproductive cycle is less with longer interbreeding intervals). Therefore, whether a species with a long lactation, and hence a long interbreeding interval, would experience a disadvantage from evolving seasonality does not depend on how long the interbreeding interval is, but whether it is out of synch with the annual cycle.
But irrespective of that question, any putative disadvantage would become less with increasing interbreeding interval.

(ii) Even if it were assumed that mothers of species with long lactations and long interbreeding intervals do not benefit from starting lactation in periods of optimal food availability, seasonally fluctuating environments might still influence neonate survival. Environmental effects on neonate survival have been demonstrated in free-ranging giraffes (Lee et al. 2017), Asian elephants (Elephas maximus) (Mumby et al. 2013) and African elephants (Loxodonta africana) (Lee et al. 2011), making the concept feasible that timing births to optimal environmental conditions may be beneficial even in these species. This factor might be even more relevant for species living in environments with more pronounced differences between seasons than the examples mentioned above. For okapi (Okapia johnstoni), a non-seasonally reproducing ruminant with a gestation period of more than 1 year, and a lactation period that rarely reaches 1 year (Bodmer and Rabb 1992), Müller et al. (2010) described a distinct seasonal neonate mortality in zoos, and modeled the consequence of restricting mating to avoid births in the two worst months of the year. In this example, population growth was not reduced by the 'lost mating opportunity' but, on the contrary, increased due to the reduction in neonate losses. At long interbirth intervals, gambling neonate survival (and hence the investment of a long gestation period) by non-seasonal births against mating opportunities may be especially relevant.

(iii) Bactrian camels have gestation periods of more than a year (Jones et al. 2009), and their lactation period is said to be up to 14-16 months (Peters and von den Driesch 1997), and they are considered seasonal long-day breeders (Skidmore 2011). For elephants, Hufenus et al. (2018) discussed evidence for long-day breeding, suggesting that this might have been particularly relevant in the proboscideans' previous distribution range that included subtropical to temperate environments. Mammoths were exposed to seasonal fluctuations in their environment (e.g., Koch et al. 1989), and probably had lactation periods and reproductive cycles similar to extant elephants (Rountrey et al. 2007; El Adli 2018). A seasonal reproduction is the default assumption in the literature, and the available evidence for mating and birth seasons supports this assumption (Rountrey et al. 2007; Fisher 2009; Rountrey et al. 2012; El Adli 2018). To date, there is no non-human mammal with a lactation period consistently longer than 1 year that lives in temperate environments, and hence, the hypothesis of van Noordwijk et al. (2013) cannot be tested conclusively.

\section{Seasonality and gestation periods}

\section{Adjusting gestation to the annual cycle}

Kiltie (1984, 1988), Owen-Smith (1988) and Owen-Smith and Ogutu (2013) explained the relevance of gestation 
periods constraining the ability of mammals to successfully compete in seasonal environments. As outlined above, if gestation periods are so long that a reproductive cycle is longer than 1 year (but not exactly 2 or 3 years), either the next reproductive cycle cannot be timed optimally in relation to the seasonal environment, leading to less favorable conditions for the offspring, or a certain period must be simply spent waiting for the next favorable time, resulting in 'lost opportunity' for reproduction. The focus on gestation period, rather than on a more comprehensive measure of a reproductive cycle, such as interbirth interval, in these considerations stems from the fact that gestation period is a stable, physiological characteristic, whereas reported interbirth intervals need not necessarily represent a species' potential, but a mixed signal integrating the biotic and abiotic factors influencing the population under observation (Sibly and Calow 1987; Ricklefs and Wikelski 2002). Additionally, gestation periods are particularly easily observed under conditions of human care, such as in zoos.

General relationships of long-day and short-day breeding and gestation period length are depicted in Fig. 3a. For long-day breeders, gestation periods should be about multiples of 1 year (or distinctively less than 6 months), and for short-day breeders, gestation periods should be either about 0.5 or 1.5 years. We are not aware of any mammals that reproduce as seasonal short-day breeders with a 1.5year gestation period. For large long-day breeders, reducing gestation period length would allow mating to occur soon after birth, resulting in the same birth date in the next cycle (Fig. 3b). For small long-day breeders, reducing gestation period length below 3 months might allow two reproductive cycles within the same favorable period (Fig. 3c). Large short-day breeders must reduce gestation below 1 year; at decreasing gestation, they increase the difference between birth and the subsequent mating period (Fig. 3d). For small short-day breeders, where gestation periods are too short to allow a mating in a window of the short-day period with environmental conditions favorable for mating, a prolongation of the gestation period may be beneficial (Jabbour et al. 1997). Typically, this does not occur via a reduced development speed of the growing embryo, but either by delayed implantation (diapause) in many groups such as certain marsupials, xenarthrans, bats, insectivorans, shrews, carnivorans, rodents and roe deer (Orr and Zuk 2014; Renfree and Fenelon 2017), or by prolonged sperm storage after mating prior to ovulation, as in certain bats (Racey and Entwistle 2000). To our knowledge, reasons why certain strategies appear limited to certain clades, e.g., why delayed implantation does not occur in primates such as the hibernating dwarf lemurs, even though delayed implantation is a conserved trait across mammals (Ptak et al. 2012), are unknown.
Seasonal reproduction as an accelerator of reproductive cycles

The gestation period length in mammals does not scale as steeply with body mass as previously thought (Hamilton et al. 2011), but is mainly influenced by phylogeny, and, hence, by not well-defined morphophysiological characteristics of species (Clauss et al. 2014). Clauss et al. (2019) suggested that species sharing the same niche should compete amongst each other by accelerating their pace of life, and showed that among extant eutherians, groups of a broadly similar niche that have comparatively shorter gestation periods are more speciose than those with comparatively longer ones. One possible mechanism for evolving shorter gestation periods may be via adaptations to seasonal reproduction. In ruminants, carnivorans and primates, relatively shorter gestation periods have been linked with a higher degree of reproductive seasonality (Zerbe et al. 2012; Heldstab et al. 2018, 2020). Similarly, Tökölyi et al. (2014) found that artiodactyls, carnivorans and rodents (but not bats or primates) have relatively shorter gestation periods in environments with more distinct temperature seasonality. When assessing eutherian gestation periods not only against body mass and litter size (as a proxy for altriciality/precociality) but also absolute latitude, latitude has a significant negative effect on gestation period (Fig. 4). This was evident even after accounting for phylogenetic affiliation (Table 1). This means that the accelerating effect of latitude on gestation period occurs both within (Zerbe et al. 2012; Heldstab et al. $2018,2020)$ and across clades, but is particularly strong across clades, supporting the notion that clades with generally shorter gestation periods are more prominent at higher latitudes.

Terrestrial and marine mammalian giants can be used to illustrate these life history concepts linked to gestation. In spite of the traditional concept of linking life history measures, such as gestation, with body mass (with increasing gestation periods at increasing mass) (Hamilton et al. 2011), the gestation period of a $3000 \mathrm{~kg}$ elephant is, at 22 months (Lüders 2018), distinctively longer than that reported for a $50,000 \mathrm{~kg}$ sperm whale (15 months) or that of a $100,000 \mathrm{~kg}$ blue whale (12 months) (Pomeroy 2011). Apparently, there are distinct clade-specific differences in measures like gestation period, such as between Afrotheria (including elephants) and Boroeutheria (including Cetartiodactyla) (Clauss et al. 2014). Additionally, seasonality of the habitat is linked to life history, with shorter gestation periods typically found in more seasonally breeding species: The food base of sperm whales are squid, hunted in total darkness at depths (Goldbogen et al. 2019) that most likely preclude an effect of season on prey population dynamics (Whitehead 1996; Best 1999). The food base of blue whales is krill with a highly seasonal bloom (Goldbogen et al. 2019). For a sperm whale, there 


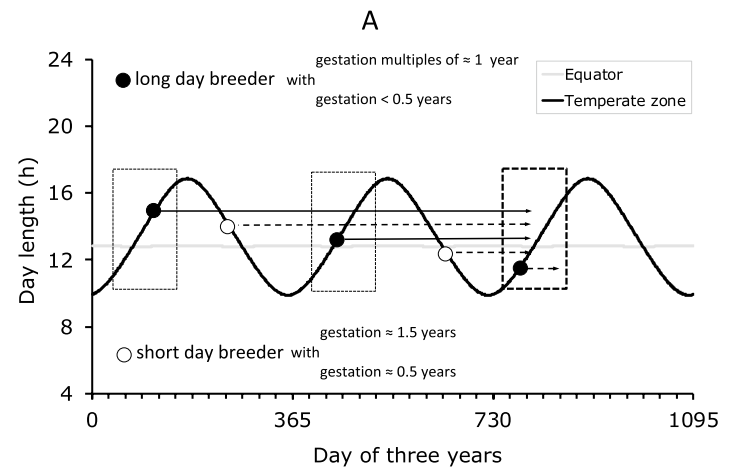

B

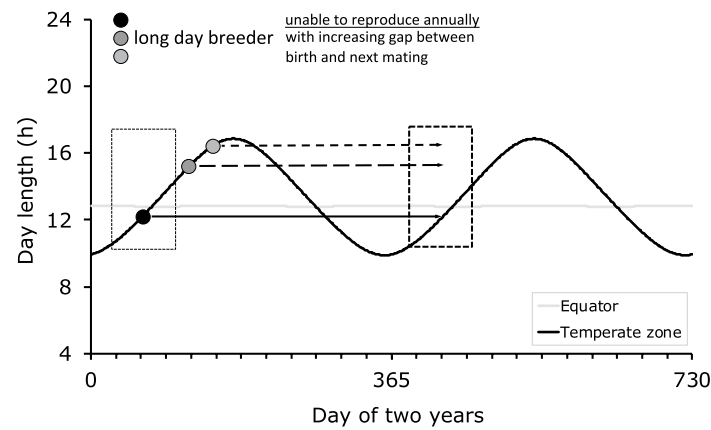

D

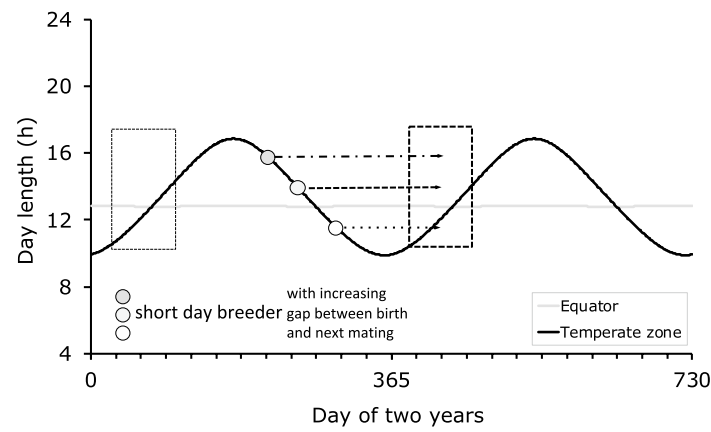

Fig. 3 Hypothetical combinations of gestation periods and photoperiod-triggered seasonal reproduction in relation to 'spring' time windows offering favorable conditions for neonate survival and resources (indicated by the box). a Typical combinations of long-day and short-day breeding in relation to a favorable birth period. b Long-day breeder giving birth in the subsequent year: benefit of shorter gestation periods. c Long-day breeder giving birth in the same year: benefit of shorter gestation periods. d Short-day breeder giving birth in

is no strong selective pressure to evolve a shorter gestation period. For a baleen whale, there should be strong selection to compress gestation to 1 year, so that the reproductive cycle is in synchrony with the annual cycle of prey availability (Lockyer 1984) and no reproductive opportunity is lost (Kiltie 1984, 1988). The shorter gestation period of the larger cetacean corresponds to this expectation. The lactation periods of the two whale species show the same pattern (Oftedal 1997), with a much shorter lactation in baleen

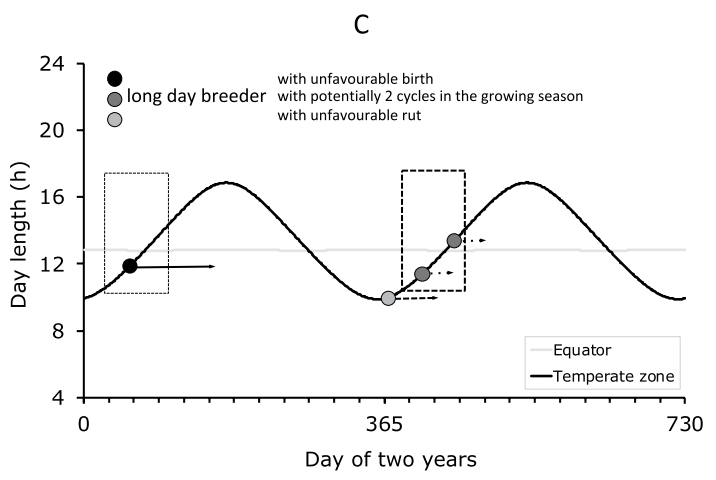

E

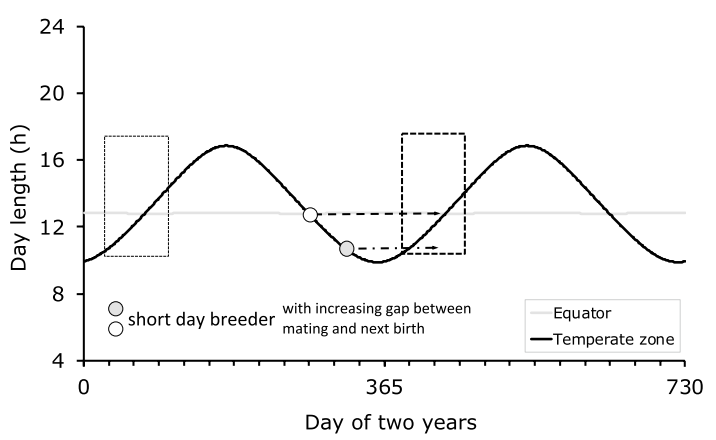

the subsequent season: increasing distance to previous birthing period by shortening gestation periods (in species whose ancestral gestation periods would have been longer, due to their body mass and phylogeny). e Short-day breeder giving birth in the subsequent season: increasing distance to birthing by increasing gestation period (in species whose ancestral gestation periods would have been shorter, due to their body mass and phylogeny)

whales that facilitates a total interbirth interval of 2 years (Lockyer 1984). Thus, although the two whale species are by magnitudes larger than the afrotherian elephants, they have shorter gestation periods, and the larger yet more seasonal of the two has the even shorter gestation period among the whales. Note that the morphophysiological adaptations that facilitate the shorter gestation periods are unknown to date, in spite of their dramatic demographic consequences in the Darwinian competition for niche occupation. Nevertheless, 
Fig. 4 Relationship between body-mass-controlled gestation length and absolute latitude in eutherian mammals. In the absence of a better proxy for the difference between precocial and altricial mammals, litter size was used, with black dots for mammals considered precocial with a litter size of about 1 , and gray dots for mammals considered altricial with larger litters. Data from Jones et al. (2009). For statistics, see Table 1

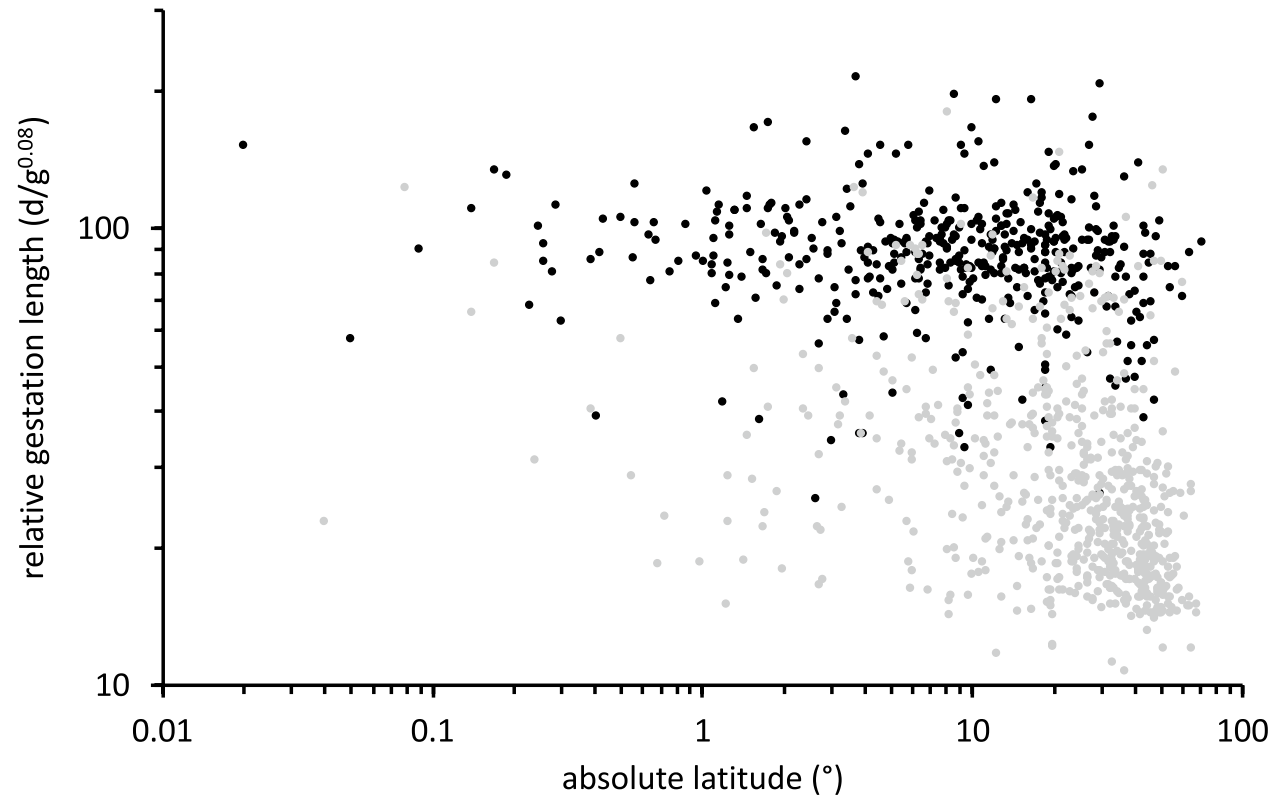

Table 1 Linear regression analysis (including 95\% confidence intervals, CI) according gestation period $=(a)$, body mass (b), litter size (c), latitude (d) using log-transformed variables throughout in gener- alized least squares (GLS) and phylogenetic generalized least squares (PGLS) in 1108 eutherian mammal species (data from Jones et al. 2009)

\begin{tabular}{|c|c|c|c|c|c|c|c|c|}
\hline \multirow[t]{2}{*}{ Statistical model } & \multicolumn{4}{|l|}{ GLS } & \multirow{2}{*}{$\begin{array}{l}\text { PGLS } \\
\text { Estimate }\end{array}$} & \multicolumn{3}{|c|}{ Max. likelihood $\lambda: 0.99(0.98-1.00)$} \\
\hline & Estimate & $95 \% \mathrm{CI}$ & $t$ & $p$ & & $95 \% \mathrm{CI}$ & $t$ & $p$ \\
\hline Intercept $(a)$ & 74 & $(68 ; 81)$ & 95.12 & $<0.001$ & 58 & $(46 ; 73)$ & 33.57 & $<0.001$ \\
\hline Body mass $[\mathrm{g}](b)$ & 0.13 & $(0.12 ; 0.13)$ & 29.46 & $<0.001$ & 0.08 & $(0.07 ; 0.10)$ & 11.85 & $<0.001$ \\
\hline Litter size $[1: 1 ; 2:>1](c)$ & -1.38 & $(-1.46 ;-1.30)$ & -33.31 & $<0.001$ & -0.16 & $(-0.24 ;-0.08)$ & -3.93 & $<0.001$ \\
\hline Absolute latitude $\left[{ }^{\circ}\right](d)$ & -0.098 & $(-0.120 ;-0.077)$ & -8.95 & $<0.001$ & -0.012 & $(-0.021 ;-0.002)$ & -2.34 & 0.019 \\
\hline
\end{tabular}

Statistics performed in R (R Core Team 2015) using packages nlme (Pinheiro et al. 2011) and caper (Orme et al. 2010), and the phylogenetic tree of Bininda-Emonds et al. (2008)

both clade-specificity and seasonality appear linked to the pace of life history.

\section{Keeping birth and mating separate}

Following the concept of Kiltie (1984, 1988), it is easy to understand why a large mammal, such as a buffalo, should evolve a 'shorter' gestation period of less than a year. However, the concept of getting reproductive cycles in synch with the annual cycle does not explain why a lemur, with a gestation period expected from body mass alone that would already easily fit into the annual cycle, should also have a shorter-than-expected gestation (Heldstab et al. 2020). To explain this observation, additional reasons for a clear separation of birthing and mating seasons need to be invoked. We are not aware of a comprehensive treatment of this question, and provide a non-exhaustive list of possible reasons.

In terms of resource allocation, rutting, mating and conception might be more beneficially placed at a time when the animals have accreted body stores, which is not at the time of birth, but at the end of the vegetative season. Especially at the initiation of lactation, females of many species experience an intense energy turnover and may experience lactational amenorrhea (Lee 1987). Depending on the species' ability to accrete body stores, which is typically higher in larger animals (Lindstedt and Boyce 1985), it may be more favorable to time the mating season prior to the final reserve accretion of the annual cycle (as in roe deer Capreolus capreolus, with a prolonged gestation due to delayed implantation) or afterwards (as in various caprids, with particularly short gestations), so that rutting and mating do not waste time that can be used for feeding during this period (Zerbe et al. 2012).

Due to the character of social interactions, the mating season may, on the one hand, be stressful or dangerous for females of certain species, and it is hence beneficial if it does not coincide with the time of raising a newborn. One example is the increased mortality risk of female cervids kept in 
zoos during the rutting period (Carisch et al. 2017). On the other hand, the presence of a male in a mating period closely after birth may make the neonate susceptible to infanticide by the new sire (Hrdy 1979; van Schaik and Janson 2000). Infanticide has been reported particularly among social species (Lukas and Huchard 2014), in primates and carnivorans, and also in sciurognath and muroid rodents (van Schaik 2000b; Lukas and Huchard 2014). The risk of infanticide is related to the weaning status of the offspring, with animals not yet weaned under particular threat (van Schaik 2000a). Therefore, avoiding infanticide, by postponing mating until after weaning, may be an important driver of shorter gestation periods in seasonal species. Infanticide occurs more frequently in non-seasonal species, where a male gains the advantage of a female becoming receptive immediately, rather than in seasonal species where the timing of the next mating period is fixed (Hrdy 1979; van Noordwijk and van Schaik 2000; Lukas and Huchard 2014).

\section{Conclusions}

Adaptations to environmental seasonality are not only important for the specific survival of a population in a certain environment, but also may be involved in the evolution of life history characteristics that determine a taxon's pace of life. Comparative approaches cannot replace experimental investigations into the physiology and determinants of seasonal organismal functions. However, comparative assays that include both specimens subjected to the conditions of their natural environment and specimens under closeto-optimal resource provision under human care have the potential to facilitate characterisations of large numbers of species, and lead to taxon-specific insights. Potential future experiments on non-photoperiodic triggers of reproduction in specimens of permissive body condition, such as temperature, humidity or secondary plant compounds, might identify species with peculiar or additional triggering mechanisms. Nevertheless, the interpretative approach of considering the reproduction of all animals to be body condition-dependent and hence possibly limited by resource supply of a natural habitat, which is alleviated under human care, with the potential additional control of a photoperiodic trigger, is a parsimonious approach that can be applied to large comparative datasets. Additionally, the daily surveillance in zoos generates high-resolution data that allow more quantitative measures of seasonal reproduction than most observations in natural habitats (such as the birth peak breadth introduced by Zerbe et al. 2012), which again facilitate the testing of hypotheses on links between reproductive seasonality and other life history characteristics. Thus, reproductive seasonality is an example of the original contribution of zoological gardens to the generation of biological knowledge beyond husbandry and management applications.

Funding Open access funding provided by University of Zurich.

\section{Compliance with ethical standards}

Conflict of interest On behalf of all authors, the corresponding author states that there is no conflict of interest.

Open Access This article is licensed under a Creative Commons Attribution 4.0 International License, which permits use, sharing, adaptation, distribution and reproduction in any medium or format, as long as you give appropriate credit to the original author(s) and the source, provide a link to the Creative Commons licence, and indicate if changes were made. The images or other third party material in this article are included in the article's Creative Commons licence, unless indicated otherwise in a credit line to the material. If material is not included in the article's Creative Commons licence and your intended use is not permitted by statutory regulation or exceeds the permitted use, you will need to obtain permission directly from the copyright holder. To view a copy of this licence, visit http://creativecommons.org/licenses/by/4.0/.

\section{References}

Best PB (1999) Food and feeding of sperm whales Physeter macrocephalus off the west coast of South Africa. Afr J Mar Sci 21:393-413

Bininda-Emonds ORP, Cardillo M, Jones KE, MacPhee RDE, Beck RMD, Grenyer R, Price SA, Vos RA, Gittleman JL, Purvis A (2008) Corrigendum: The delayed rise of present-day mammals. Nature 456:274

Blanco MB, Rasoazanabary E, Godfrey LR (2015) Unpredictable environments, opportunistic responses: reproduction and population turnover in two wild mouse lemur species (Microcebus rufus and M. griseorufus) from eastern and western Madagascar. Am J Primatol 77:936-947

Bodmer RE, Rabb GB (1992) Okapia johnstoni. Mamm Species 422:1-8

Boyd IL (2000) State-dependent fertility in pinnipeds: contrasting capital and income breeders. Funct Ecol 14:623-630

Brockman DK, van Schaik CP (2005) Seasonality and reproductive function. In: Brockman DK, van Schaik CP (eds) Seasonality in primates: studies of living and extinct human and non-human primates. Cambridge University Press, Cambridge, pp 269-305

Bronson FH (1989) Mammalian reproductive biology. University of Chicago Press, Chicago

Bronson FH (2009) Climate change and seasonal reproduction in mammals. Philos Trans R Soc B 364:3331-3340

Bronson F, Heideman P (1994) Seasonal regulation of reproduction in mammals. In: Knobil E, Neill JD (eds) The physiology of reproduction. Raven Press, New York, pp 541-584

Carisch L, Müller DWH, Hatt J-M, Bingaman Lackey L, Rensch EE, Clauss M, Zerbe P (2017) Seasonal mortality in zoo ruminants. Zoo Biol 36:74-86

Celik O, Aydin S, Celik N, Yilmaz M (2015) Peptides: basic determinants of reproductive functions. Peptides 72:34-43

Clauss M, Kienzle E, Wiesner H (2003) The botanical, structural and chemical composition of different pelleted feeds used in a captive browsing ruminant, the moose (Alces alces). In: Fidgett AL, 
Clauss M, Gansloßer U, Hatt JM, Nijboer J (eds) Zoo animal nutrition, vol II. Filander Verlag, Fürth, pp 91-101

Clauss M, Dittmann MT, Müller DWH, Zerbe P, Codron D (2014) Low scaling of a life history variable: analysing eutherian gestation periods with and without phylogeny-informed statistics. Mamm Biol 79:9-16

Clauss M, Müller DWH, Codron D (2019) Within-niche pace of life acceleration as a fundamental evolutionary principle: a mammal pilot test case. Evol Ecol Res 20:385-401

Delgadillo JA, De La Torre-Villegas S, Arellano-Solis V, Duarte G, Malpaux B (2011) Refractoriness to short and long days determines the end and onset of the breeding season in subtropical goats. Theriogenology 76:1146-1151

D'Occhio MJ, Baruselli PS, Campanile G (2019) Influence of nutrition, body condition, and metabolic status on reproduction in female beef cattle: a review. Theriogenology 125:277-284

El Adli J (2018) Reproductive life histories of mammoths. PhD thesis, University of Michigan

Fisher DC (2009) Paleobiology and extinction of proboscideans in the Great Lakes region of North America. In: Haynes G (ed) American megafaunal extinctions at the end of the Pleistocene. Springer, Dordrecht, pp 55-75

Goldbogen JA, Cade DE, Wisniewska DM, Potvin J, Segre PS, Savoca MS, Hazen EL, Czapanskiy MF, Kahane-Rapport SR, DeRuiter SL, Gero S (2019) Why whales are big but not bigger: physiological drivers and ecological limits in the age of ocean giants. Science 366:1367-1372

Hamilton MJ, Davidson AD, Sibly RM, Brown JH (2011) Universal scaling of production rates across mammalian lineages. Proc $\mathrm{R}$ Soc B 278:560-566

Hansen PJ (1985) Photoperiodic regulation of reproduction in mammals breeding during long days versus mammals breeding during short days. Anim Reprod Sci 9:301-315

Heldstab SA, Müller DWH, Graber SM, Bingaman Lackey L, Rensch E, Hatt J-M, Zerbe P, Clauss M (2018) Geographical origin, delayed implantation and induced ovulation explain reproductive seasonality in the Carnivora. J Biol Rhythms 33:402-419

Heldstab SA, van Schaik CP, Müller DWH, Rensch E, Bingaman Lackey L, Zerbe P, Hatt J-M, Clauss M, Matsuda I (2020) Reproductive seasonality in primates: patterns, concepts and unsolved questions. Biol Rev. https://doi.org/10.1111/brv.12646

Hrdy SB (1979) Infanticide among animals: a review, classification, and examination of the implications for the reproductive strategies of females. Ethol Sociobiol 1:13-40

Hufenus R, Schiffmann C, Hatt J-M, Müller DWH, Bingaman Lackey L, Clauss M, Zerbe P (2018) Seasonality of reproduction in elephants (Elephas maximus and Loxodonta africana): underlying photoperiodic cueing? Mamm Rev 48:261-276

Jabbour H, Hayssen V, Bruford M (1997) Conservation of deer: contributions from molecular biology, evolutionary ecology, and reproductive physiology. J Zool 243:461-484

Janson C, Verdolin J (2005) Seasonality of primate births in relation to climate. In: Brockman DK, van Schaik CP (eds) Seasonality in primates: studies of living and extinct human and non-human primates. Cambridge University Press, Cambridge, pp 307-350

Jones KE, Bielby J, Cardillo M, Fritz SA, O’Dell J, Orme CDL, Safi K, Sechrest W, Boakes EH, Carbone C, Connolly C, Cutts MJ, Foster JK, Grenyer R, Habib M, Plaster CA, Price SA, Rigby EA, Rist J, Teacher A, Bininda-Emonds ORP, Gittleman JL, Mace GM, Purvis A (2009) PanTHERIA: a species-level database of life history, ecology, and geography of extant and recently extinct mammals. Ecology 90:2648

Jönsson KI (1997) Capital and income breeding as alternative tactics of resource use in reproduction. Oikos 78:57-66

Kanamori T, Kuze N, Bernard H, Malim TP, Kohshima S (2010) Feeding ecology of Bornean orangutans (Pongo pygmaeus morio) in
Danum Valley, Sabah, Malaysia: a 3-year record including two mast fruitings. Am J Primatol 72:820-840

Kerby J, Post E (2013) Capital and income breeding traits differentiate trophic match-mismatch dynamics in large herbivores. Philos Trans R Soc B 368:20120484

Kiltie RA (1984) Seasonality, gestation time, and large mammal extinctions. In: Martin PS, Klein RG (eds) Quaternary extinctions: a prehistoric revolution. University of Arizona Press, Tucson, pp 299-314

Kiltie RA (1988) Gestation as a constraint on the evolution of seasonal breeding in mammals. In: Boyce MS (ed) Evolution of life histories of mammals: theory and patterns. Yale University Press, New Haven, pp 257-289

Koch PL, Fisher DC, Dettman D (1989) Oxygen isotope variation in the tusks of extinct proboscideans: a measure of season of death and seasonality. Geology 17:515-519

Lechner-Doll M, Deutsch A, Lang D (2000) Nutritional management of ungulates in captivity-should we learn from natural seasonality of the vegetation? In: Nijboer J, Hatt J, Kaumanns W, Beijnen A, Ganslosser U (eds) Zoo animal nutrition. Filander, Fürth, pp 205-212

Lee PC (1987) Nutrition, fertility and maternal investment in primates. J Zool 213:409-422

Lee PC, Lindsay WK, Moss CJ (2011) Ecological patterns of variability in demographic rates. In: Moss CJ, Croze H, Lee PC (eds) The Amboseli elephants. University of Chicago Press, Chicago, pp 74-88

Lee DE, Bond ML, Bolger DT (2017) Season of birth affects juvenile survival of giraffe. Popul Ecol 59:45-54

Lewis RJ, Kappeler PM (2005) Are Kirindy sifaka capital or income breeders? It depends. Am J Primatol 67:365-369

Lindstedt SL, Boyce MS (1985) Seasonality, fasting endurance, and body size in mammals. Am Nat 125:873-878

Lithner S, Jonsson KI (2002) Abundance of owls and bramblings Fringilla montifringilla in relation to mast seeding in southeastern Sweden. Ornis Svecica 12:35-45

Lockyer C (1984) Review of baleen whale (Mysticeti) reproduction and implications for management. Rep Int Whal Comm Spec Issue 6:27-50

Lüders I (2018) Elephant pregnancy and parturition: normal and abnormal. In: Miller RE, Laberski N, Calle P (eds) Fowler's zoo and wild animal medicine. Current therapy, vol 9. Elsevier, St. Louis, pp 96.91-96.99

Lukas D, Huchard E (2014) The evolution of infanticide by males in mammalian societies. Science 346:841-844

Müller DWH, Müller SI, Bingaman Lackey L, Hammer C, Hammer S, Leus K, Maier M, Hatt J-M, Clauss M (2010) Less can be more: considering seasonal differences of newborn mortality in breeding regimes. Proc Int Conf Dis Zoo Wild Anim 2:96-98

Mumby HS, Courtiol A, Mar KU, Lummaa V (2013) Climatic variation and age-specific survival in Asian elephants from Myanmar. Ecology 94:1131-1141

Nicholls TJ, Jackson GL, Follett BK (1989) Reproductive refractoriness in the Welsh Mountain ewe induced by a short photoperiod can be overridden by exposure to a shorter photoperiod. Biol Reprod 40:81-86

Oftedal OT (1997) Lactation in whales and dolphins: evidence of divergence between baleen-and toothed-species. J Mammary Gland Biol Neoplasia 2:205-230

Ogutu JO, Piepho HP, Dublin HT, Bhola N, Reid RS (2010) Rainfall extremes explain interannual shifts in timing and synchrony of calving in topi and warthog. Popul Ecol 52:89-102

Ogutu JO, Owen-Smith N, Piepho HP, Dublin HT (2015) How rainfall variation influences reproductive patterns of African savanna ungulates in an equatorial region where photoperiod variation is absent. PLoS One 10:e0133744 
Orme D, Freckleton R, Thomas G, Petzoldt T, Fritz S, Isaac N (2010) Caper: comparative analyses of phylogenetics and evolution in R. R package version 0.4/r71. See http://caper.r-forge.r-proje ct.org/

Orr TJ, Zuk M (2014) Reproductive delays in mammals: an unexplored avenue for post-copulatory sexual selection. Biol Rev 89:889-912

Owen-Smith RN (1988) Megaherbivores - the influence of very large body size on ecology. Cambridge University Press, Cambridge

Owen-Smith N, Ogutu JO (2013) Controls over reproductive phenology among ungulates: allometry and tropical-temperate contrasts. Ecography 36:256-263

Paul MJ, Zucker I, Schwartz WJ (2008) Tracking the seasons: the internal calendars of vertebrates. Philos Trans R Soc B 363:341-361

Peters J, von den Driesch A (1997) The two-humped camel (Camelus bactrianus): new light on its distribution, management and medical treatment in the past. J Zool 242:651-679

Pinheiro J, Bates D, DebRoy S, Sarkar D, R Development Core Team (2011) nlme: linear and nonlinear mixed effects models. R package version 3. 1-102. https://cran.r-project.org/web/packages/ nlme/

Pomeroy P (2011) Reproductive cycles of marine mammals. Anim Reprod Sci 124:184-193

Prendergast BJ (2005) Internalization of seasonal time. Horm Behav 48:503-511

Ptak GE, Tacconi E, Czernik M, Toschi P, Modlinski JA, Loi P (2012) Embryonic diapause is conserved across mammals. PLoS One 7:e33027

R Core Team (2015) R: a language and environment for statistical computing. R Foundation for Statistical Computing, Vienna, Austria. http://www.R-project.org/

Racey PA, Entwistle AC (2000) Life-history and reproductive strategies of bats. In: Chrichton EG, Kutsch P (eds) The reproductive biology of bats. Academic Press, New York, pp 363-414

Renfree MB, Fenelon JC (2017) The enigma of embryonic diapause. Development 144:3199-3210

Ricklefs RE, Wikelski M (2002) The physiology/life-history nexus. Trends Ecol Evol 17:462-468

Robinson JE, Wayne NL, Karsch FJ (1985) Refractoriness to inhibitory day lengths initiates the breeding season of the Suffolk ewe. Biol Reprod 32:1024-1030

Rountrey AN, Fisher DC, Vartanyan S, Fox DL (2007) Carbon and nitrogen isotope analyses of a juvenile woolly mammoth tusk: evidence of weaning. Quat Int 169:166-173

Rountrey AN, Fisher DC, Tikhonov AN, Kosintsev PA, Lazarev PA, Boeskorov G, Buigues B (2012) Early tooth development, gestation, and season of birth in mammoths. Quat Int 255:196-205

Sempéré AJ, Blanvillain C, Mauget R, Lacroix A, Chemineau P (1995) Effects of melatonin implantation or artificial long days on seasonal ovulatory activity in roe deer (Capreolus capreolus). Anim Reprod Sci 38:127-136

Sibly RI, Calow PE (1987) Ecological compensation-a complication for testing life-history theory. J Theor Biol 125:177-186
Skidmore JA (2011) Reproductive physiology in female old world camelids. Anim Reprod Sci 124:148-154

Solonen T (2005) Breeding of the Tawny Owl Strix aluco in Finland: responses of a southern colonist to the highly variable environment of the North. Ornis Fennica 82:97-106

Stearns SC (1992) The evolution of life histories. Oxford University Press, Oxford

Stephens PA, Boyd IL, McNamara JM, Houston AI (2009) Capital breeding and income breeding: their meaning, measurement, and worth. Ecology 90:2057-2067

Tissier ML, Réale D, Garant D, Bergeron P (2020) Consumption of red maple in anticipation of beech mast-seeding drives reproduction in Eastern chipmunks. J Anim Ecol 89:1190-1201

Tökölyi J, Schmidt J, Barta Z (2014) Climate and mammalian life histories. Biol J Linn Soc 111:719-736

van Noordwijk MA, van Schaik CP (2000) Reproductive patterns in eutherian mammals: adaptations against infanticide? In: van Schaik CP, Janson CH (eds) Infanticide by males and its implications. Cambridge University Press, Cambridge, pp 322-360

van Noordwijk MA, Willems EP, Atmoko SSU, Kuzawa CW, van Schaik CP (2013) Multi-year lactation and its consequences in Bornean orangutans (Pongo pygmaeus wurmbii). Behav Ecol Sociobiol 67:805-814

van Schaik CP (2000a) Infanticide by male primates: the sexual selection hypothesis revisited. In: van Schaik CP, Janson CH (eds) Infanticide by males and its implications. Cambridge University Press, Cambridge, pp 27-60

van Schaik CP (2000b) Vulnerability to infanticide by males: patterns among mammals. In: van Schaik CP, Janson CH (eds) Infanticide by males and its implications. Cambridge University Press, Cambridge, pp 61-71

van Schaik CP, Janson CH (eds) (2000) Infanticide by males and its implications. Cambridge University Press, Cambridge

van Schaik CP, van Noordwijk MA (1985) Interannual variability in fruit abundance and the reproductive seasonality in Sumatran long-tailed macaques (Macaca fascicularis). J Zool 206:533-549

Visser ME, Caro SP, Van Oers K, Schaper SV, Helm B (2010) Phenology, seasonal timing and circannual rhythms: towards a unified framework. Philos Trans R Soc B 365:3113-3127

Whitehead H (1996) Variation in the feeding success of sperm whales: temporal scale, spatial scale and relationship to migrations. J Anim Ecol 65:429-438

Zerbe P, Clauss M, Codron D, Bingaman Lackey L, Rensch E, Streich WJ, Hatt J-M, Müller DWH (2012) Reproductive seasonality in captive wild ruminants: implications for biogeographical adaptation, photoperiodic control, and life history. Biol Rev 87:965-990

Publisher's Note Springer Nature remains neutral with regard to jurisdictional claims in published maps and institutional affiliations. 